\title{
Quasi-Resonant Interconnects: A Low Power, Low Latency Design Methodology
}

\author{
Jonathan Rosenfeld and Eby G. Friedman, Fellow, IEEE
}

\begin{abstract}
Design and analysis guidelines for quasi-resonant interconnect networks (QRN) are presented in this paper. The methodology focuses on developing an accurate analytic distributed model of the on-chip interconnect and inductor to obtain both low power and low latency. Excellent agreement is shown between the proposed model and SpectraS simulations. The analysis and design of the inductor, insertion point, and driver resistance for minimum power-delay product is described. A case study demonstrates the design of a quasi-resonant interconnect, transmitting a $5 \mathrm{~Gb} / \mathrm{s}$ data signal along a $5 \mathrm{~mm}$ line in a TSMC 0.18- $\mu \mathrm{m}$ CMOS technology. As compared to classical repeater insertion, an average reduction of $91.1 \%$ and $37.8 \%$ is obtained in power consumption and delay, respectively. As compared to optical links, a reduction of $97.1 \%$ and $35.6 \%$ is observed in power consumption and delay, respectively.
\end{abstract}

Index Terms-Latency, on-chip inductors, on-chip interconnects, power dissipation, resonance.

\section{INTRODUCTION}

A PRIMARY challenge in high performance, high complexity integrated circuits (ICs) is the on-chip interconnect [1]. Transmitting clock, data, and communications signals over large die areas requires long interconnections among the various circuit modules. Consequently, as technology scales, the interconnect cross section decreases while operating frequencies increase. The impact of these trends on high performance systems is significant. Long interconnects with smaller cross sections exhibit increased capacitance and resistance, resulting in greater power consumption, latency, and signal attenuation. Furthermore, wire inductance can no longer be ignored due to high signal frequencies and long wire lengths. Two figures of merit that determine the length of interconnect in which inductance effects are significant have been described in [2].

Some of these challenges are evident in the international technology roadmap for semiconductors (ITRS) [1], as shown in Fig. 1. According to ITRS predictions, the total on-chip wire length will increase linearly with technology, reaching above $2000 \mathrm{~m} / \mathrm{cm}^{2}$ by the end of this decade [see Fig. 1(a)]. This trend supports the assumption that long interconnects will be signifi-

Manuscript received March 09, 2007; revised September 13, 2007. Current version published January 14, 2009. This work was supported in part by the National Science Foundation under Contract CCF-0541206, by grants from the New York State Office of Science, Imaging Systems, and by grants from Intel Corporation, Eastman Kodak Company, and Freescale Semiconductor Corporation.

The authors are with the Department of Electrical and Computer Engineering, University of Rochester, Rochester, NY 14627-0231 USA (e-mail: rosenfel@ece.rochester.edu; friedman@ece.rochester.edu).

Digital Object Identifier 10.1109/TVLSI.2008.2011197

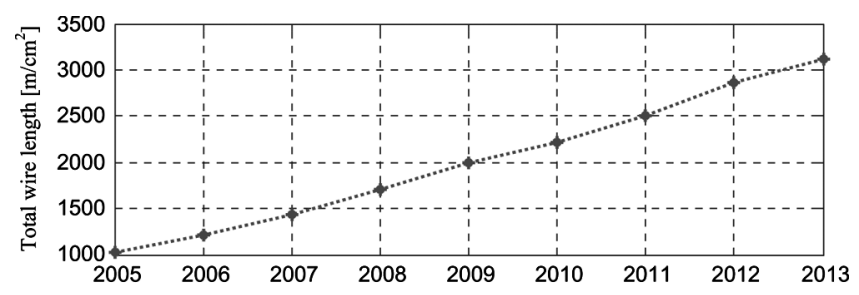

(a)

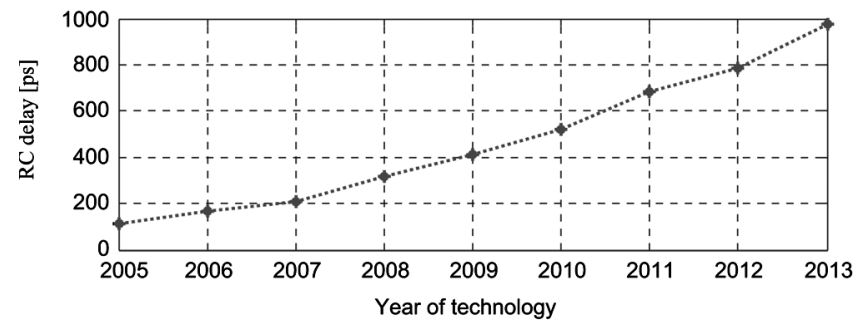

(b)

Fig. 1. 2005 ITRS predictions: (a) total interconnect length of metal 1 and 5 intermediate levels; (b) $R C$ interconnect delay for a $1 \mathrm{~mm} \mathrm{Cu}$ global wire.

cant in future technologies. A prediction for resistance-capacitance $(R C)$ interconnect delay is depicted in Fig. 1(b). The $R C$ delay increases quadratically with technology, reaching above $500 \mathrm{ps}$ (for a $1 \mathrm{~mm} \mathrm{Cu}$ global wire) by 2010 . This trend supports the assumption that long interconnects with small cross sections exhibit increasing latency and delay uncertainty.

To combat these phenomena, traditional repeater insertion methods have been widely developed [3]. Unfortunately, as interconnect lengths increase, a larger number of repeaters is required. This results in significant power dissipation, increased delay, and larger area. Low power techniques, therefore, in the form of low swing signaling, have been developed. In [4], current mode signaling in an ultra-low voltage environment is used to transmit high data rate signals. In this approach, a current sense amplifier at the far end of the line detects a current difference and converts it into a voltage difference. To improve both delay and energy dissipation, a transmitter generating a differential current detected by a current mode sense amplifier at the receiving end has been proposed in [5]. To accommodate differential operation, redundant circuitry is used.

To minimize static power dissipation associated with current mode signaling, an adaptive bandwidth bus architecture based on hybrid current voltage mode repeaters for long $R C$ interconnect has been proposed in [6]. In this approach, the interconnect is divided into smaller segments. Appropriately spaced repeaters amplify the signal and drive the subsequent interconnect segment. An adaptive control unit is placed at the input 
of the line and connected to a control line, which is common to all of the repeater stages. Contrary to the current mode signaling approach, the authors of [7] and [8] suggest the use of low voltage signaling over long on-chip interconnects. In [7], a heuristic algorithm for buffer insertion that considers noise, delay, and power is proposed. The method proposed in [8] is based on the so-called swing limited interconnect accelerator. This circuit has a three stage cascade inverter configuration with keepers. The keepers limit the voltage on the interconnect to permit low swing operation. Additional inverters are placed at the output to restore the signal level to full swing.

A different approach exploiting wire inductance at high frequencies is introduced in [9]. Chang et al. [9] suggest that at lower frequencies the resistive part of the interconnect is dominant, behaving as a distributed $R C$ network. At higher frequencies, the inductive component of the transmission line dominates, behaving as a distributed inductance-capacitance $(L C)$ network. In this scheme, a $1-\mathrm{GHz}$ link operating with phase shift keying modulation on a $7.5-\mathrm{GHz}$ sinusoidal carrier is presented. This type of modulation, however, results in relatively large power dissipation and poor spectral efficiency. Alternatively, the authors of [10] propose mitigating the dispersion due to high frequencies by utilizing a return to zero (RZ) signaling scheme in which sharp current pulses transmit data. The transmitted data is modulated to higher frequencies, maximizing the effect of the wire inductance. This approach allows the interconnects to behave in a relatively dispersionless fashion.

As an alternative to electrical interconnects and related electrical limitations, optoelectronic links [11] have been considered. Optical interconnections promise to achieve high bandwidth by providing high density parallel communication channels. The interface between the electrical and optical signals however is a major issue in optoelectronic applications.

In this paper, a low power, low latency on-chip interconnect design methodology is proposed. The methodology is based on inserting an on-chip spiral inductor to resonate the interconnect around the fundamental harmonic of the transmitted signal. In this way, the interconnect capacitance resonates with the inserted on-chip inductance. The fundamental harmonic of the input signal is amplified and transmitted to the output. This approach lowers power consumption, since the energy resonates between the electric and magnetic fields rather than dissipates as heat. Consequently, buffers are eliminated, significantly reducing power consumption and signal latency.

This paper is organized into six sections. The use of on-chip resonance in integrated circuits as well as the principle of resonance for data transmission are presented in Section II. The interconnect and spiral inductor models are described in Section III. In Section IV, a quasi-resonant interconnect design methodology is described, followed by a case study demonstrating 5 Gbps data signal distribution along a $5-\mathrm{mm}$ interconnect in Section V. Simulation results and a comparison to other schemes are presented in Section VI. Finally, some conclusions are offered in Section VII. In Appendix A, a receiver circuit is described. The $A B C D$ matrices are reviewed in Appendix B and a derivation of the $50 \%$ delay is provided in Appendix C.

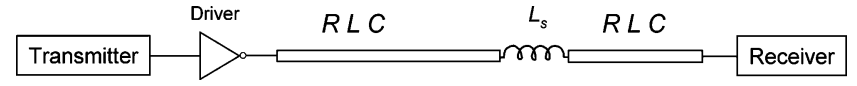

Fig. 2. Quasi-resonant network.

\section{PRINCIPLE OF Quasi-Resonant InterconNects}

Following the invention of the telegraph and the telephone, transmission techniques were developed to accommodate the increasing distance between the transmitter and receiver while supporting a higher transmission rate. Pupin introduced the concept of inserting reactance sources in series with long telegraphic lines in 1899 [12], [13]. Pupin suggested inserting inductance coils in a network composed of a uniform conductor with a telephonic transmitter and receiver. The inductance coils are inserted in series at periodically recurring locations. The electrical line parameters and the distance between the coils are adjusted to reduce signal attenuation, enabling transmission of speech waves over very long distances. The application of transmission lines with periodic inductors to microwave applications is described in [14], and is based on Pupin's invention.

The concept of exploiting standing waves in transmission lines was first introduced by Chi in 1994 [15]. A global resonant clock distribution network was later introduced in 2003 by Chan et al. [16]. In this circuit, a set of discrete on-chip spiral inductors and capacitors is attached to a traditional H-tree structure. The capacitance of the clock distribution network resonates with the inductance, while the on-chip capacitors establish a mid-rail dc voltage around which the grid oscillates. This approach lowers the power consumption, skew, and jitter.

Chueh et al. [17] designed and evaluated a two-phase resonant clock generation and distribution system with layout extracted inductor parameters in a $0.13-\mu \mathrm{m}$ CMOS process. The circuit includes a programmable replenishing clock generator and tunable capacitors. Both skew and jitter are reduced with this approach.

A design methodology for resonant clock distribution networks is presented in [18]. Design guidelines based on an accurate distributed transmission model are proposed, supporting low power $\mathrm{H}$-tree clock distribution networks. The design methodology provides tradeoffs among the operating frequency, the size of the on-chip inductors and capacitors, and the output resistance of the driving buffer. A sensitivity analysis of resonant $\mathrm{H}$-tree clock distribution networks is also provided.

The proposed quasi-resonant interconnect network architecture is illustrated in Fig. 2. The transmitter at the near end of the interconnect modulates the input data signal. The signal modulation supports a quasi-periodic signal with a single resonant frequency. The transmitter is followed by an inverter driving the interconnect. The $R L C$ distributed transmission line is separated by an on-chip spiral inductor $L_{s}$ inserted at a specific point to resonate the network at a desired frequency for minimum power consumption and delay. The receiver at the far end of the interconnect demodulates the transmitted signal back into the original input bit stream.

Since the fundamental harmonic of the input signal is amplified by the magnitude of the transfer function, the network 
resonates at a specific target frequency and magnitude. This behavior is required to transfer a full swing sinusoidal signal at the resonance frequency to the far end of the interconnect. To satisfy this objective, consider the output signal in the frequency domain

$$
V_{\text {out }}(s)=H^{\prime}(s) \cdot V_{\text {in }}(s)
$$

where $H^{\prime}(s)$ is the transfer function of the network between the transmitter and the receiver (including the driver), and $V_{\text {in }}(s)$ is the input data bit. The input data signal in the time domain can be represented by a Fourier series, assuming $V_{\text {in }}(s)$ is a periodic signal

$$
v_{\text {in }}(t)=\sum_{k=-\infty}^{\infty} a_{k} e^{j k \omega_{p} t}
$$

where $a_{k}$ is the $k^{\text {th }}$ harmonic of the signal and $\omega_{p}$ is the resonant radian frequency. In the case of a periodic square waveform with period time $t_{p}$, transition time $t_{t}$, and amplitude $V_{\mathrm{dd}}$, the fundamental harmonic (positive and negative) is

$$
a_{ \pm 1}= \pm \frac{V_{\mathrm{dd}} t_{p}}{2 t_{t} \pi^{2}}\left(e^{-j \omega_{p} t_{t}}-1\right) .
$$

Equation (1) implies that the required magnitude of the transfer function is

$$
\left|H^{\prime}\left(j \omega_{p}\right)\right|=\frac{\left(\frac{V_{\mathrm{dd}}}{2}\right)}{\left(2\left|a_{1}\right|\right)}=\frac{V_{\mathrm{dd}}}{4\left|a_{1}\right|} .
$$

Substituting (3) into (4) results in

$$
\left|H^{\prime}\left(j \omega_{p}\right)\right|=\frac{t_{t} \pi^{2}}{t_{p} \sqrt{8\left(1-\cos \left(\omega_{p} t_{t}\right)\right)}} .
$$

Equation (5) describes the magnitude of the transfer function at $\omega_{p}$ to transfer a full swing waveform. For example, at a $5-\mathrm{GHz}$ operating frequency $\left(t_{p}=200 \mathrm{ps}\right)$ and $t_{t}=20 \mathrm{ps}$, the magnitude of the transfer function is 0.8 .

\section{INTERCONNECT AND SPIRAL INDUCTOR MODELS}

Accurate models of the quasi-resonant network are presented in this section. Transmission line and on-chip spiral inductor models are described in Section III-A and III-B, respectively.

\section{A. Interconnect Model}

As data signal frequencies exceed the multigigahertz regime, distributed models of interconnects are required to accurately incorporate high frequency effects into the system behavior. The wire inductance can no longer be excluded from the model and traveling wave reflections should be characterized by a distributed structure. Using a classical distributed model, an incremental section of line length $\Delta z$ is modeled as a lumped element circuit. In this representation, $R, L$, and $C$ are the resistance, inductance, and capacitance per unit length, respectively. The lumped resistance represents the lossy component of the transmission line.

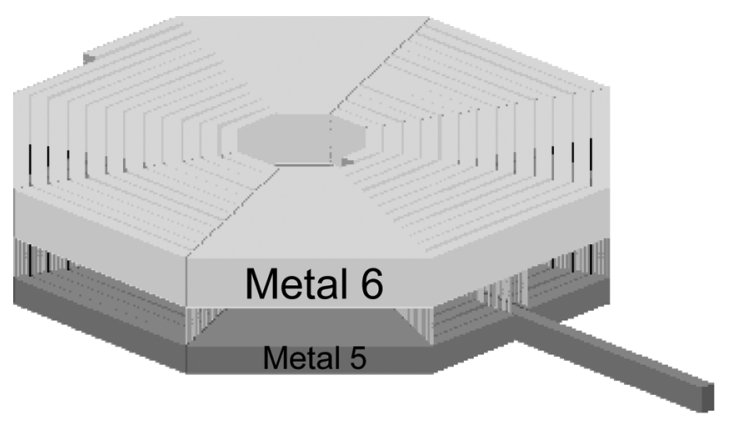

(a)

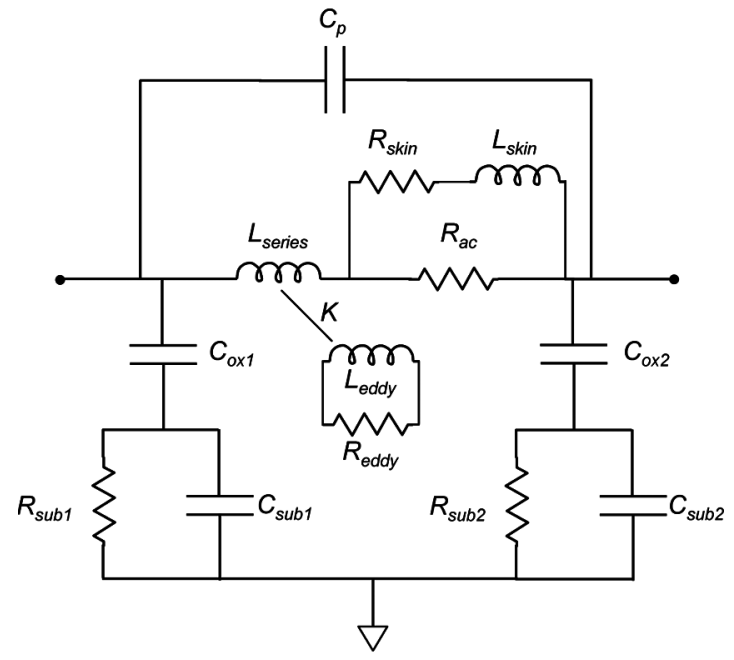

(b)

Fig. 3. Model of an on-chip spiral inductor: (a) structure of an octagonal on-chip spiral inductor; (b) lumped model of a spiral inductor.

\section{B. On-Chip Spiral Inductor Model}

To accurately account for the parasitic effects of the on-chip spiral inductor, a thirteen lumped element model is used. The physical structure and a lumped model of the on-chip inductor are illustrated in Fig. 3(a) and (b), respectively. Note that the octagonal on-chip spiral inductor shown in Fig. 3(a) utilizes Metal 5 and Metal 6 where the two layers are connected by vias. In this manner, the inductor can achieve a higher $\mathrm{Q}$, as analyzed by SPIRAL, a 3-D spiral inductor design and synthesis tool. ${ }^{1}$

The capacitance $C_{p}$ represents the capacitive coupling between the windings of the spiral inductor. The elements $L_{\text {series }}$ and $R_{\mathrm{ac}}$ represent the inductance and parasitic resistance, respectively, while $R_{\text {skin }}$ and $L_{\text {skin }}$ model the skin effect. Also note that $L_{\text {series }}$ incorporates the eddy current effect coupled to the inductor by the coefficient $K$. The parasitic capacitance between the lines and the substrate is modeled by $C_{\mathrm{ox}}$. The parallel $C_{\text {sub }}$ and $R_{\text {sub }}$ combination models the parasitic resistance and capacitance to the substrate.

\section{Design Methodology}

In this section, the quasi-resonant interconnect design methodology is described. The design of the transmitter and

${ }^{1}$ [Online]. Available: http://www.oea.com. 


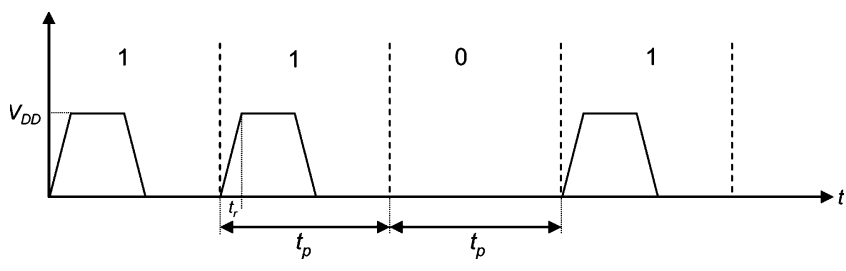

Fig. 4. Example of transmitting a "1011" bit stream.

receiver is presented in Section IV-A and IV-B, respectively. The input impedance and transfer function of the quasi-resonant network are analytically characterized in Section IV-C. Based on these expressions, a power consumption model followed by a closed-form analytic expression are developed in Section IV-D. In Section IV-E, closed-form analytic expressions for the signal delay are described. Finally, design guidelines for the quasi-resonant interconnect that minimizes the power-delay product is described in Section IV-F.

\section{A. Transmitter Design}

Proper operation of the network requires resonance at a single target frequency. As illustrated in Fig. 4, the return to zero (RZ) amplitude shift keying modulation scheme is chosen to support the single transmission frequency of the input data. In this scheme, the data is transferred at $1 / t_{p}$ bits/s.

This scheme has three advantages when applied to the proposed quasi-resonant interconnect methodology. The transmitted signal has a single frequency (with amplitude variation, i.e., $V_{\mathrm{dd}}$ and $0 \mathrm{~V}$ to distinguish between logic one and zero, respectively) designed to match the resonance frequency of the network. Power is dissipated only during transmission of logic one, and only during half of the time period. Finally, no complex circuitry is required to produce this modulation scheme.

The transmitter generates the required modulated signal which is amplified by the buffer chain at the input of the driver (see Fig. 2). The proposed modulation scheme, transmitter circuit, transistor level circuit, and simulated signal waveforms are illustrated in Fig. 5.

To realize the modulation scheme shown in Fig. 5(a), an AND operation is performed between the clock and the input data followed by a buffer chain, as shown in Fig. 5(b). The high frequency data rate supported by the quasi-resonant interconnects requires a high speed transmitter and receiver. To maintain high speed operation, a transmission gate-based circuit (consisting of transistors $\mathrm{M}_{\mathrm{t} 1}, \mathrm{M}_{\mathrm{t} 2}, \mathrm{M}_{\mathrm{t} 3}$, and $\mathrm{M}_{\mathrm{t} 4}$ ) is used for the logic, as shown in Fig. 5(c). In the case where both the clock and data are at logic one, the transmission gate $\mathrm{M}_{\mathrm{t} 1}$ passes the logic one state to the input of the buffer chain, while the inverter (consisting of transistors $\mathrm{M}_{\mathrm{t} 3}$ and $\mathrm{M}_{\mathrm{t} 4}$ ) turns off transmission gate $\mathrm{M}_{\mathrm{t} 2}$. In all of the other cases, the logic zero state is passed to the input of the buffer chain.

Transistor $\mathrm{M}_{\mathrm{t} 5}$ restores the voltage (equal to $V_{\mathrm{th}}$ ) associated with the operation of transmission gates $\mathrm{M}_{\mathrm{t} 1}$ and $\mathrm{M}_{\mathrm{t} 2}$. The input data as well as the modulated signal are shown in Fig. 5(d). In this example, a "100100111" bit stream is modulated at a $5-\mathrm{GHz}$ operating frequency. Note that the modulated signal follows the

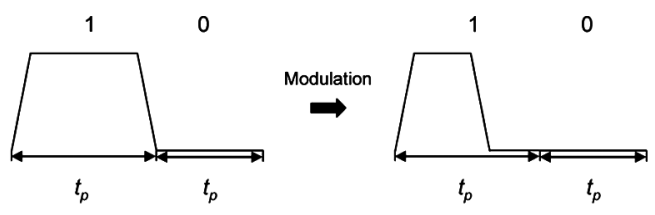

(a)

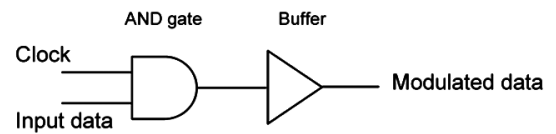

(b)

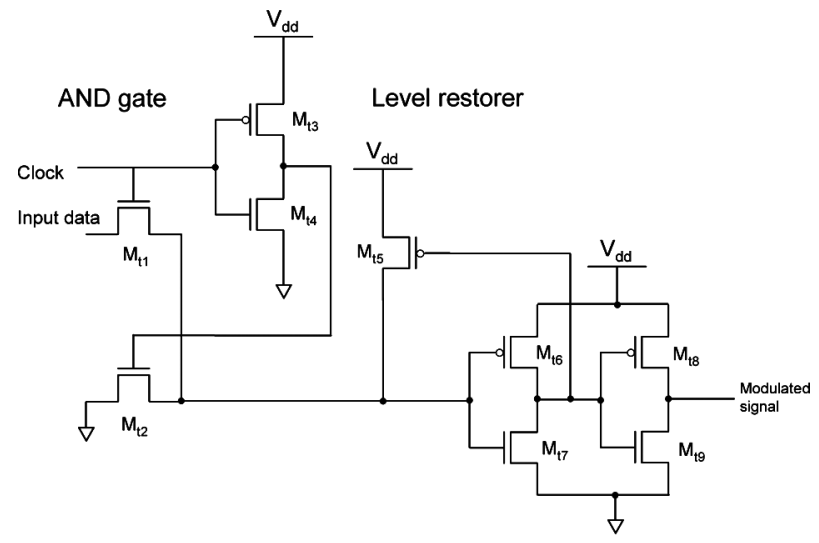

(c)
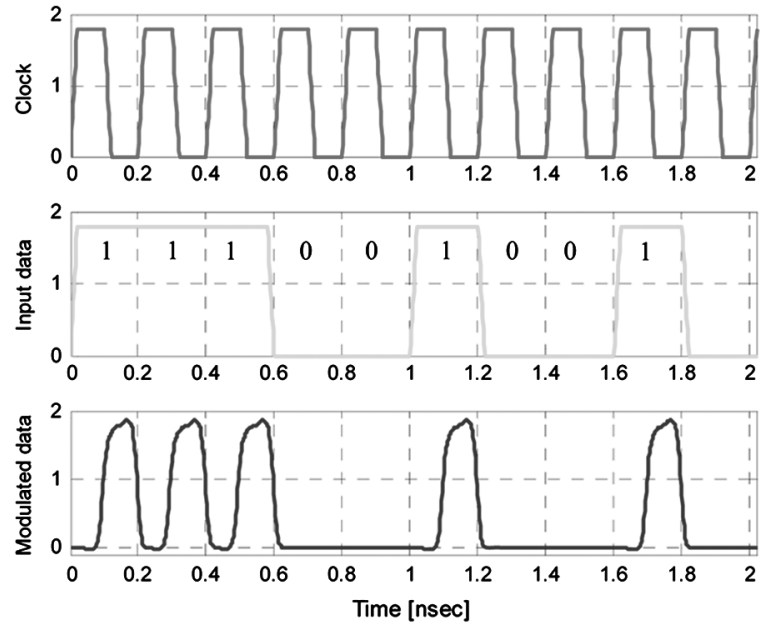

(d)

Fig. 5. Transmitter circuit: (a) modulation scheme; (b) gate level circuit; (c) transistor level circuit; (d) signal waveforms.

return to zero amplitude shift keying scheme, essential for quasiresonant operation.

\section{B. Receiver Design}

The receiver located at the far end of the interconnect (see Fig. 2) demodulates the transmitted data into the original signal. A logic level description of the proposed receiver circuit, a transistor level circuit, and simulated signal waveforms are depicted in Fig. 6. The principle of the demodulation scheme is based on a sample and hold $(\mathrm{S} / \mathrm{H})$ operation, as shown in Fig. 6(a). When the clock is high, the switch closes and the data is sampled and transferred. The sampled signal charges (and discharges) the parasitic capacitance $C_{p}$. When the clock is low, the switch 


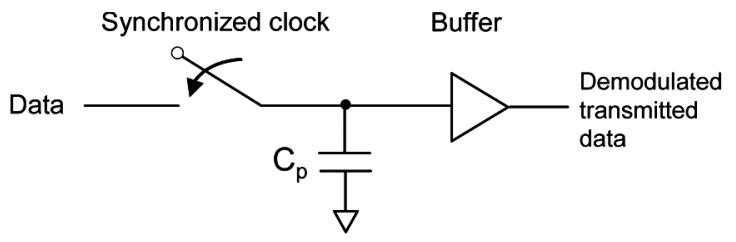

(a)

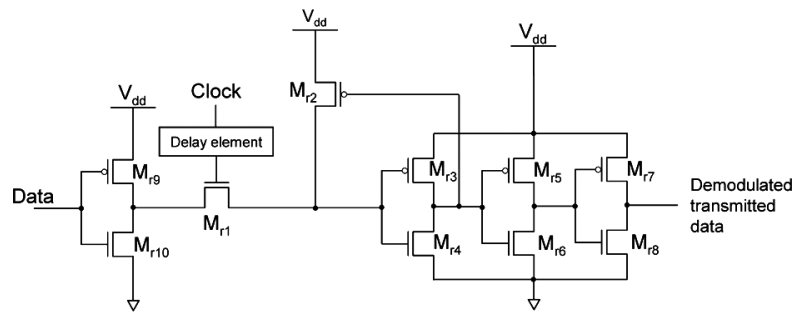

(b)
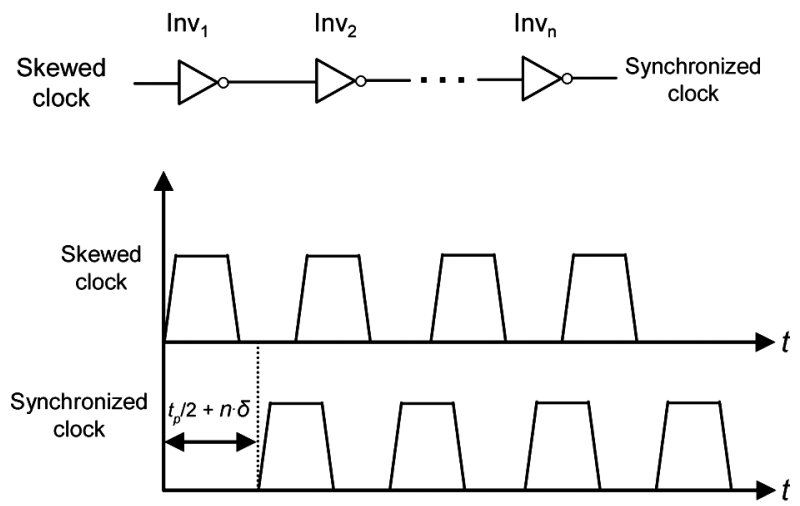

(c)
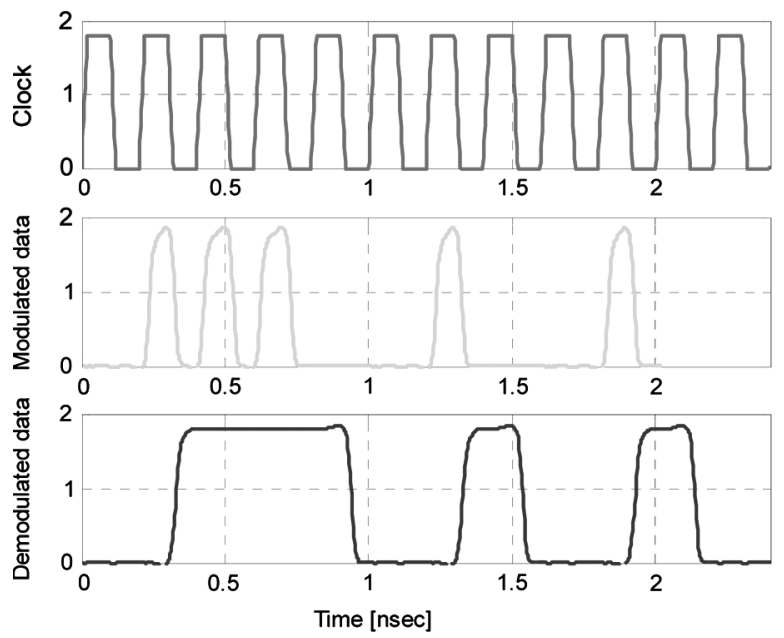

(d)

Fig. 6. Receiver circuit: (a) sample and hold circuit; (b) transistor level circuit; (c) delay element circuit and timing diagram; (d) signal waveforms.

is open and the logic state is stored (or held) until the following clock cycle. In this manner, the $\mathrm{S} / \mathrm{H}$ circuit prolongs the duration of the high state signals over the entire clock cycle $t_{p}$ without altering the duration of the low state signals.

The receiver circuit at the transistor level is shown in Fig. 6(b). In this circuit, when the clock state is high, transistor $\mathrm{M}_{\mathrm{r} 1}$ turns on and the data is sampled and transferred. When

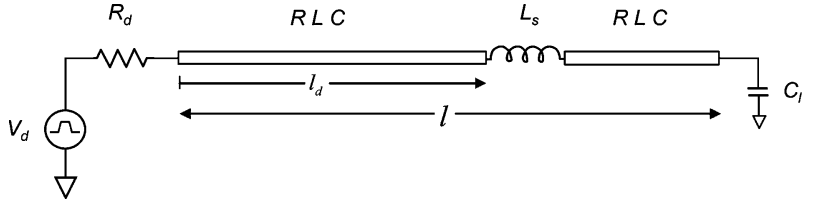

Fig. 7. Quasi-resonant network.

the data state is low, the restorer transistor $\mathrm{M}_{\mathrm{r} 2}$ turns on, maintaining the high state signal at the input of the second inverter (consisting of transistors $\mathrm{M}_{\mathrm{r} 3}$ and $\mathrm{M}_{\mathrm{r} 4}$ ). This mechanism serves a dual purpose. It restores the voltage associated with the transmission gate $\mathrm{M}_{\mathrm{r} 1}$, and prevents charge leakage by replenishing the charge on the parasitic capacitance of transistors $\mathrm{M}_{\mathrm{r} 3}$ and $\mathrm{M}_{\mathrm{r} 4}$ (through the feedback connection). When the clock logic is low, $M_{\mathrm{r} 2}$ prevents the charge from leaking. When the data logic is high, transistor $\mathrm{M}_{\mathrm{r} 2}$ turns off and the logic low state is transferred to the output. Finally, when the clock state is low, the previous logic state is preserved until the following clock cycle.

Note that for the receiver to operate properly, the allowed skew between the clock and the incoming modulated data should be less then one quarter of the clock cycle. This constraint is required since the demodulation circuit is level sensitive. To synchronize the clock with the data, a delay element is used, as depicted in Fig. 6(b). The delay of the arriving modulated data is determined from simulations or the analytic delay expressions presented in Section IV-E. A delay element based on inverters is used as shown in Fig. 6(c). The delay element provides a course and a fine delay. If course tuning is required (i.e., the delay of a half clock cycle, $t_{p} / 2$ ), an odd number of inverters is used. If fine tuning is required (i.e., the intrinsic delay of the inverters), an even number of inverters is required. In this manner, for an odd number of inverters, a total delay of $t_{p} / 2+n \delta$ is achieved. For an even number of inverters, a total delay of $n \delta$ is achieved. $\delta$ and $n$ are the intrinsic delay of a single inverter and number of inverters, respectively.

Simulation results of this circuit are presented in Fig. 6(d). In this example, the output of the modulation circuit shown in Fig. 5(c) drives the demodulation circuit shown in Fig. 6(b). As depicted in Fig. 6(d), the output waveform is identical to the input data waveforms illustrated in Fig. 5(d). An alternative receiver circuit that does not require the interconnect and inverter delay element is presented in Appendix A.

\section{Network Input Impedance and Transfer Function}

To obtain analytic closed-form expressions of the power consumption and delay of the quasi-resonant interconnect, the input impedance and the transfer function of the network are determined in this subsection. The quasi-resonant network (QRN) (between the transmitter and receiver) including the driver is shown in Fig. 7. Note that the driver is modeled as a linearized voltage source $V_{d}$ serially connected with a driver resistance $R_{d}$. The load of the interconnect is modeled as a capacitor $C_{l}$.

To analyze this type of structure, an accurate analytic model is developed based on $A B C D$ parameters. From transmission line 
theory, the $A B C D$ matrix for the entire structure is a product of the individual matrices

$$
\left[\begin{array}{ll}
A & B \\
C & D
\end{array}\right]=M_{\mathrm{int} 1} \cdot M_{s} \cdot M_{\mathrm{int} 2} \cdot M_{l}
$$

where $M_{\mathrm{int} 1}, M_{s}, M_{\mathrm{int} 2}$, and $M_{l}$ are the $A B C D$ matrix of the first interconnect section, the on-chip inductor [based on the model of Fig. 3(b)], the second interconnect section, and the load capacitance, respectively. These matrices are described in Appendix B. Matrices $M_{\mathrm{int} 1}$ and $M_{\mathrm{int} 2}$ are based on the distributed transmission line model presented in Section III-A. From the overall $A B C D$ parameters, the transfer function $H(j \omega)$ (excluding the driver resistance) and the input impedance $Z_{\text {in }}(j \omega)$ of the system are, respectively

$$
\begin{aligned}
H(j \omega) & =\frac{1}{A} \\
Z_{\text {in }}(j \omega) & =R_{\text {in }}+j X_{\text {in }}=\frac{A}{C} .
\end{aligned}
$$

The transfer function of the overall network shown in Fig. 7 (including the driver resistance) is given by (9), where $R_{d}$ and $R_{\text {in }}$ are the driver and input resistance, respectively, and $X_{\text {in }}$ is the input reactance

$$
\begin{aligned}
\left|H^{\prime}(j \omega)\right| & =\left|\frac{Z_{\text {in }}}{Z_{\text {in }}+R_{d}}\right| \cdot|H(j \omega)| \\
& =\left|\frac{R_{\text {in }}+j X_{\text {in }}}{\left(R_{\text {in }}+R_{d}\right)+j X_{\text {in }}}\right| \cdot|H(j \omega)| \\
& =\sqrt{\frac{R_{\text {in }}^{2}+X_{\text {in }}^{2}}{\left(R_{d}+R_{\text {in }}\right)^{2}+X_{\text {in }}^{2}}} \cdot|H(j \omega)| .
\end{aligned}
$$

The driver resistance is extracted from (9). Equating $\left|H^{\prime}(j \omega)\right|$ to the value obtained from (5) at a specific frequency $\omega_{p}$ and solving for $R_{d}$ results in

$$
R_{d}=\left(\sqrt{\frac{\left|H\left(j \omega_{p}\right)\right|^{2}}{\left|H^{\prime}\left(j \omega_{p}\right)\right|^{2}} \cdot\left(R_{\text {in }}^{2}+X_{\text {in }}^{2}\right)-X_{\text {in }}^{2}}\right)-R_{\text {in }}
$$

where $H^{\prime}\left(j \omega_{p}\right)$ and $H\left(j \omega_{p}\right)$ are given by (5) and (7), respectively.

\section{Power Consumption Model}

The average power consumed by the network shown in Fig. 1 is

$$
P_{\text {total }, \text { avg }}=P_{\text {trx }, \text { avg }}+P_{\text {qrn,avg }}+P_{\text {rec }, \text { avg }}
$$

where $P_{\text {trx,avg }}$ and $P_{\text {rec,avg }}$ are the average power consumption of the transmitter and receiver, respectively, and $P_{\mathrm{qrn}, \text { avg }}$ is the average power consumption of the quasi-resonant network (including the driver).

The resonant interconnect network is a passive linear network. A one-port network, as depicted in Fig. 8, can therefore be used to determine $P_{\text {qrn,avg }}$. The output impedance of the driver $R_{d}$ and the input impedance of the network $Z_{\text {in }}$ determine the power consumption of the network.

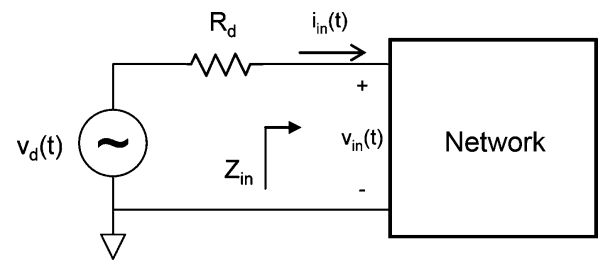

Fig. 8. One-port network driven by a voltage source.

Power, defined as the rate at which energy is absorbed [20], is

$$
P_{\text {qrn,avg }}=\frac{1}{2} V_{\text {in,rms }}^{2} \cdot \Re\left\{\frac{1}{Z_{\text {in }}}\right\}
$$

where $V_{\text {in,rms }}$ is the effective or root-mean square value of any periodic voltage and is

$$
V_{\mathrm{in}, \mathrm{rms}}=\left(\frac{1}{T} \int_{t_{0}}^{t_{0}+T}\left|v_{\mathrm{in}}(t)\right|^{2} d t\right)^{1 / 2}
$$

where $T$ is the time period of the periodic function $v_{\text {in }}(t)$. The input voltage $v_{\text {in }}(t)$ (see Fig. 8) can be expressed in terms of the voltage source function $v_{d}(t)$, and is

$$
v_{\text {in }}(t)=v_{d}(t) \cdot \frac{Z_{\text {in }}}{Z_{\text {in }}+R_{d}} .
$$

Substituting (13) and (14) into (12) results in

$$
\begin{aligned}
P_{\text {qrn }, \text { avg }} & =\frac{1}{2} V_{\text {rms }, d}^{2} \cdot\left|\frac{Z_{\text {in }}}{Z_{\text {in }}+R_{d}}\right|^{2} \cdot \Re\left\{\frac{1}{Z_{\text {in }}}\right\} \\
& =\frac{1}{2} V_{\text {rms }, d}^{2} \cdot \frac{R_{\text {in }}}{\left(R_{\text {in }}+R_{d}\right)^{2}+X_{\text {in }}^{2}} .
\end{aligned}
$$

Substituting (10) into (15) yields a simplified expression, depending solely on the input impedance of the network $Z_{\text {in }}$

$$
P_{\mathrm{qrn}, \mathrm{avg}}=\frac{1}{2} V_{\mathrm{rms}, d}^{2} \cdot\left|\frac{H^{\prime}\left(j \omega_{p}\right)}{H\left(j \omega_{p}\right)}\right|^{2} \cdot \frac{R_{\mathrm{in}}}{\left(R_{\mathrm{in}}^{2}+X_{\mathrm{in}}^{2}\right)} .
$$

\section{E. Signal Delay Model}

The total signal delay of the network shown in Fig. 2 is

$$
t_{d, \mathrm{tot}}=t_{d, \mathrm{trx}}+t_{d, \mathrm{qrn}}+t_{d, \mathrm{rec}}
$$

where $t_{d, \text { trx }}$ and $t_{d, \text { rec }}$ are the transmitter and receiver signal delay, respectively, and $t_{d, \mathrm{qrn}}$ is the signal delay of the quasiresonant network (including the driver).

A derivation of a closed-form expression for $t_{d, \mathrm{qrn}}$ is based on the work of Chen and Friedman [21]. Chen and Friedman [21] based their analysis on a Fourier series, where a time-domain waveform as well as the $50 \%$ delay is approximated by the summation of several sinusoids. Although this method is designed for interconnects driven by a periodic signal, it can also be applied to a quasi-resonant network. As shown in Fig. 4, the QRN is driven by a modulated signal periodic in time with two different symbols for logic one and zero. It can therefore be assumed that a quasi-resonant network is driven by a quasi-periodic signal. 
The $50 \%$ delay can be expressed as

$$
t_{d, \mathrm{qrn}}=\frac{\arctan x_{0}}{\omega_{p}}-\frac{t_{r}}{2}
$$

where $x_{0}$ is a single real root of a third-order polynomial, as described in Appendix C. Note that the value of $\arctan x_{0}$ is in the range of $[0, \pi]$; otherwise, $\pi$ should be added or subtracted from $x_{0}$. In the case of three real roots, the output waveform is not shaped like a square wave and can no longer represent a logic state. Note that the derivation of (18) assumes that the 50\% delay is less than $t_{p} / 2-t_{r} / 2$.

\section{F. Design Guideline}

The QRN design process is summarized as a flow diagram in Fig. 9. The interconnect geometries, i.e., length, width, and thickness, are designed according to technological constraints and requirements. Once the geometry and transmission frequency are specified, the next step is to determine the line resistance, inductance, and capacitance per unit length. A lookup table for different on-chip inductor magnitudes and the corresponding equivalent lump model parameters (such as depicted in Fig. 3) can be extracted from experimental measurements or an electromagnetic field solver. ${ }^{1}$

Expressions (16) and (18), developed in Section IV-C to IV-E, are used to optimize the QRN to minimize the power-delay product. Simultaneously solving (16) and (18) for different on-chip inductances and insertion points along the interconnect results in a power-delay product which can be described graphically. The equivalent driver resistance is determined from (10). The required magnitude of the on-chip inductor, insertion point, and driver resistance to enable optimum power-delay operation are inferred from these graphs.

If the on-chip inductance, insertion point, and driver resistance are not physically realizable, the design cycle is repeated with a different technology, transmission frequency, or interconnect geometry. Once the parameters are determined, the driver is designed based on the specified output resistance. Finally, the transmitter is designed based on the operating frequency and driver input capacitance.

\section{CAse Study}

The design guidelines and principles presented in Section IV and illustrated in Fig. 9 are demonstrated in a case study. This example is based on a TSMC 0.18- $\mu \mathrm{m}$ CMOS technology, transmitting data at a $5 \mathrm{~Gb} / \mathrm{s}$ frequency. The target time period is $t_{p}=$ $200 \mathrm{ps}$ and the rise (fall) time is $t_{r}=20 \mathrm{ps}$ with a supply voltage $V_{\mathrm{dd}}=1.8 \mathrm{~V}$.

The on-chip octagonal inductor model parameters are extracted from SPIRAL. ${ }^{1}$ The spiral inductors (varying between 1 to $10 \mathrm{nH}$ ) have been designed and optimized to achieve a maximum $\mathrm{Q}$ at $5 \mathrm{GHz}$.

The layout geometry and configuration of the quasi-resonant network are schematically illustrated in Fig. 10. To reduce crosstalk and coupling noise from neighboring interconnects, the quasi-resonant interconnect is shielded by two parallel

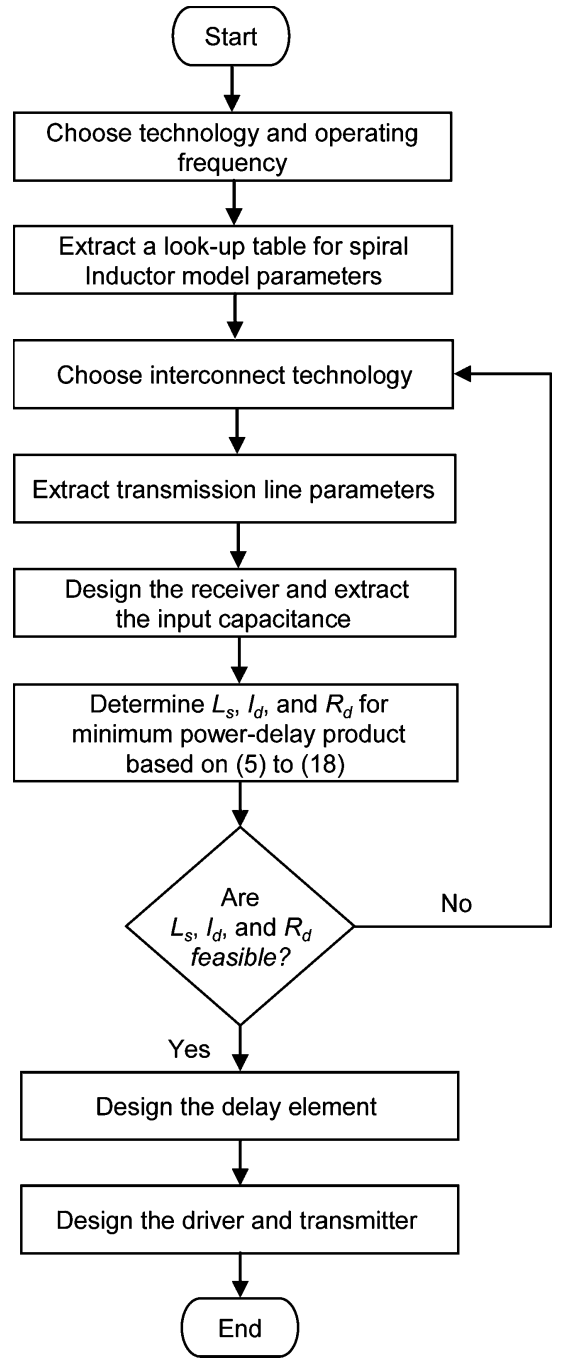

Fig. 9. Flow diagram of QRN design process.

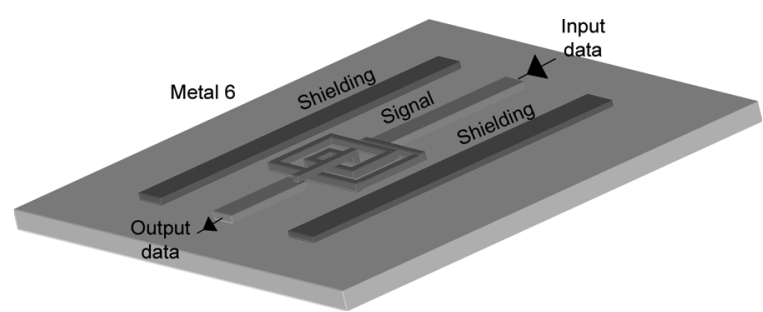

Fig. 10. Layout of a resonant transmission line network.

ground lines. The shield lines reduce the parasitic coupling capacitance between the two signal lines. Another strategy to mitigate inductive crosstalk is to utilize perpendicular lines on different metal layers.

The separation between the signal and ground lines is $2 \mu \mathrm{m}$. The width of the signal and ground lines is 2 and $4 \mu \mathrm{m}$, respectively, while the thickness of each of the lines is $1 \mu \mathrm{m}$. In this example, the interconnect parameters (including the shield lines) are $l=5 \mathrm{~mm}, R=17 \mathrm{~m} \Omega / \mu \mathrm{m}, L=1.66 \mathrm{pH} / \mu \mathrm{m}$, and $C=0.072 \mathrm{fF} / \mu \mathrm{m}$. 
TABLE I

TRANSISTOR WIDTH OF THE RECEIVER AND TRANSMITTER CiRCUITS, $L=0.18 \mu \mathrm{m}$

\begin{tabular}{l|l|l|r}
\hline \multicolumn{4}{c}{ Width [ $\mu \mathrm{m}]$} \\
\hline \multicolumn{2}{c}{ Receiver } & \multicolumn{2}{c}{ Transmitter } \\
\hline $\mathrm{M}_{\mathrm{r} 1}$ & 2.0 & $\mathrm{M}_{\mathrm{t} 1}$ & 1.0 \\
\hline $\mathrm{M}_{\mathrm{r} 2}$ & 1.0 & $\mathrm{M}_{\mathrm{t} 2}$ & 1.0 \\
\hline $\mathrm{M}_{\mathrm{r} 3}$ & 1.5 & $\mathrm{M}_{\mathrm{t} 3}$ & 2.5 \\
\hline $\mathrm{M}_{\mathrm{r} 4}$ & 2.0 & $\mathrm{M}_{\mathrm{t} 4}$ & 1.0 \\
\hline $\mathrm{M}_{\mathrm{r} 5}$ & 2.5 & $\mathrm{M}_{\mathrm{t} 5}$ & 0.5 \\
\hline $\mathrm{M}_{\mathrm{r} 6}$ & 1.0 & $\mathrm{M}_{\mathrm{t} 6}$ & 2.5 \\
\hline $\mathrm{M}_{\mathrm{r} 7}$ & 2.5 & $\mathrm{M}_{\mathrm{t} 7}$ & 1.0 \\
\hline $\mathrm{M}_{\mathrm{r} 8}$ & 1.0 & $\mathrm{M}_{\mathrm{t} 8}$ & 0.3 \\
\hline $\mathrm{M}_{\mathrm{r} 9}$ & 7.5 & $\mathrm{M}_{\mathrm{t} 9}$ & 0.2 \\
\hline $\mathrm{M}_{\mathrm{r} 10}$ & 3.0 & & \\
\hline
\end{tabular}

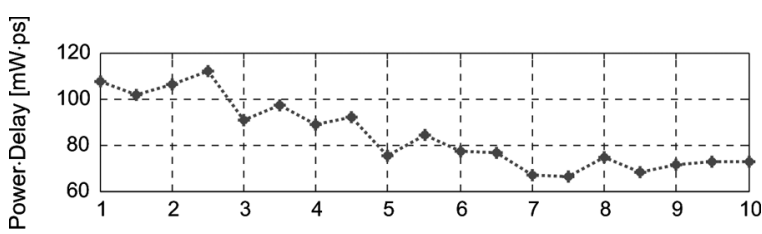

(a)

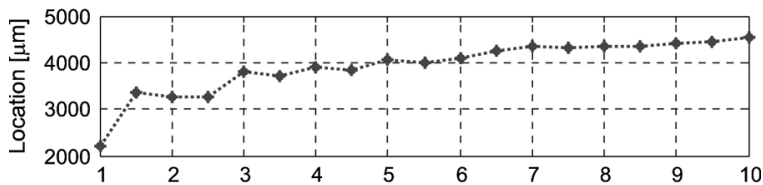

(b)

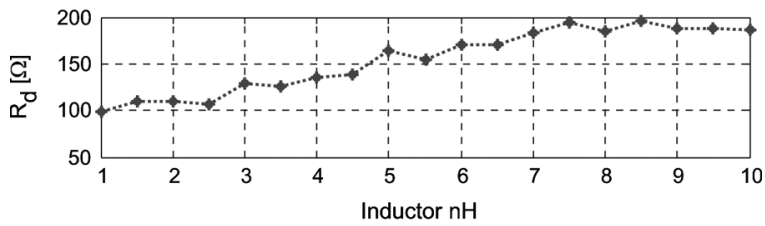

(c)

Fig. 11. Design example of a 5-mm-long interconnect operating at a 5-Gb/s transmission frequency: (a) minimum power-delay product as a function of inductance; (b) insertion location as a function of inductance; and (c) driver resistance as a function of inductance.

The receiver is based on the topology proposed in Fig. 6(b) and the transistor widths are listed in Table I. The input capacitance of the receiver circuit [i.e., the first buffer shown in Fig. 6(b)] is $C_{l}=17.4 \mathrm{fF}$.

Once the capacitive load of the interconnect is known, $L_{s}$, $l_{d}$, and $R_{d}$ are determined to minimize power and delay. The minimum power-delay product as a function of the on-chip inductance, using (5) to (18), is shown in Fig. 11(a). The corresponding insertion point and driver resistance as a function of the on-chip inductance are shown in Figs. 5(b) and (c), respectively. As evident from Fig. 11, the minimum power-delay product occurs when an inductor $L_{s}=7.5 \mathrm{nH}$, inserted at $l_{d}=$ $4.3 \mathrm{~mm}$, and a driver resistance of $R_{d}=195 \Omega$ are used. For these parameters, the power consumption and signal delay of the QRN are shown in Fig. 12.

The magnitude and phase of the QRN transfer function are depicted in the frequency domain, in Fig. 13. Good agreement between simulations and the proposed analytic expressions for the magnitude and phase of the transfer function is achieved,

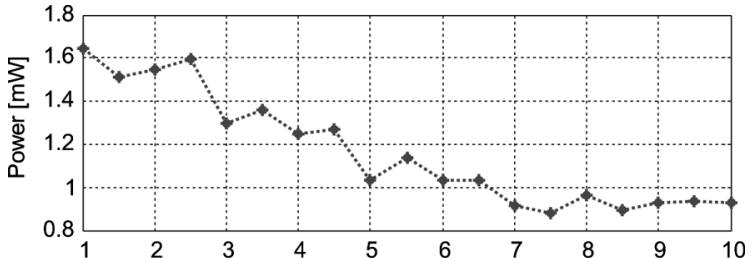

(a)

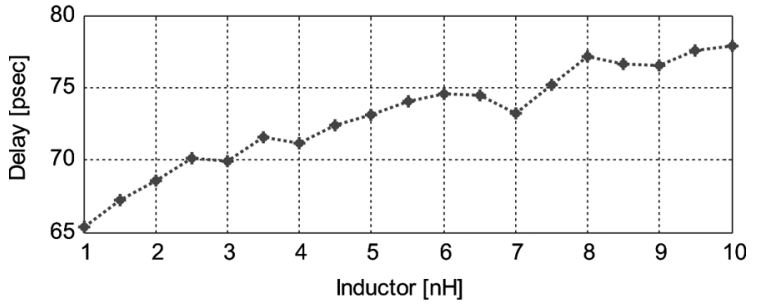

(b)

Fig. 12. Power and delay: (a) power consumption; (b) signal delay.

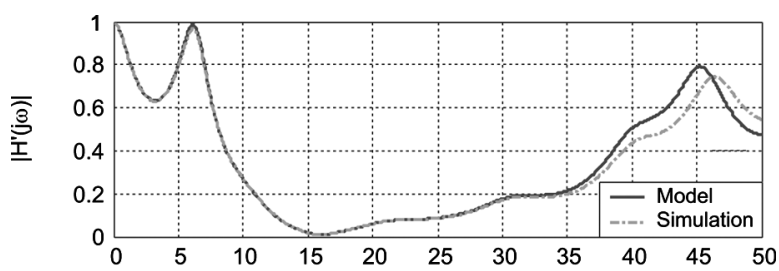

(a)

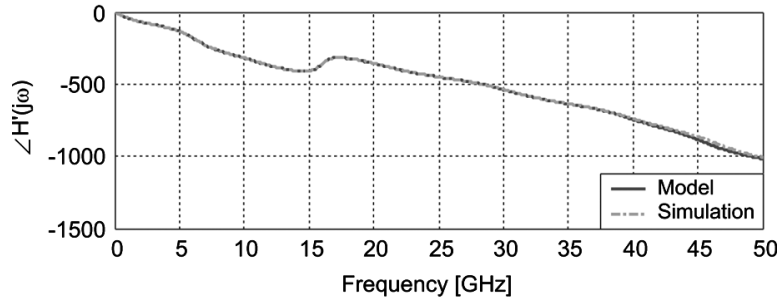

(b)

Fig. 13. Frequency response of the transfer function: (a) magnitude; (b) phase.

exhibiting a maximum error of $19.5 \%$ and $2.8 \%$, respectively. At a 5-GHz frequency, the magnitude of the transfer function is near resonance, here described as quasi-resonance. This behavior occurs since the addition of the parasitic capacitance of the on-chip spiral inductor reduces the resonant frequency, i.e., $1 / 2 \pi(L C)^{1 / 2}$. The resonance peak, therefore, shifts to a higher frequency. Note that the magnitude of the transfer function reaches 0.8 at a $5-\mathrm{GHz}$ frequency, as determined from (5).

The real and imaginary parts of the network input impedance are shown in Fig. 14. With an on-chip inductor $L_{s}=7.5 \mathrm{nH}$ operating at $5 \mathrm{GHz}$, the input impedance of the network is $Z_{\text {in }}=$ $110+j 55$. Good agreement between simulations and the proposed analytic expressions for the magnitude and phase of the input impedance are achieved, exhibiting a maximum error of $4.3 \%$ and $37 \%$, respectively.

The effect of the on-chip inductor on the magnitude of the transfer function is shown in Fig. 15. In this example, the magnitude of the transfer function reaches 0.8 with a 7.5-nH on-chip 
TABLE II

Simulated And ANalytic Power Consumption And Delay of THe Receiver, Transmitter, AND QRN

\begin{tabular}{l|c|c|c|c|c|c|c}
\hline & \multicolumn{2}{|c|}{ Simulation } & \multicolumn{2}{c|}{ QRN } & \multicolumn{3}{c}{ Total } \\
\hline & Receiver & Transmitter & Model & Simulation & Model & Simulation & $\begin{array}{c}\text { Error } \\
\%\end{array}$ \\
\hline $\begin{array}{l}\text { Power } \\
{[\mathrm{mW}]}\end{array}$ & 0.45 & 0.34 & 0.88 & 1.18 & 1.67 & 1.90 & 12.11 \\
\hline $\begin{array}{l}\text { Delay } \\
{[\mathrm{psec}]}\end{array}$ & 27.50 & 86.00 & 74.00 & 80.00 & 187.50 & 193.50 & 3.10 \\
\hline
\end{tabular}

TABLE III

COMPARISON OF QRN MODEL WITH SIMULATION

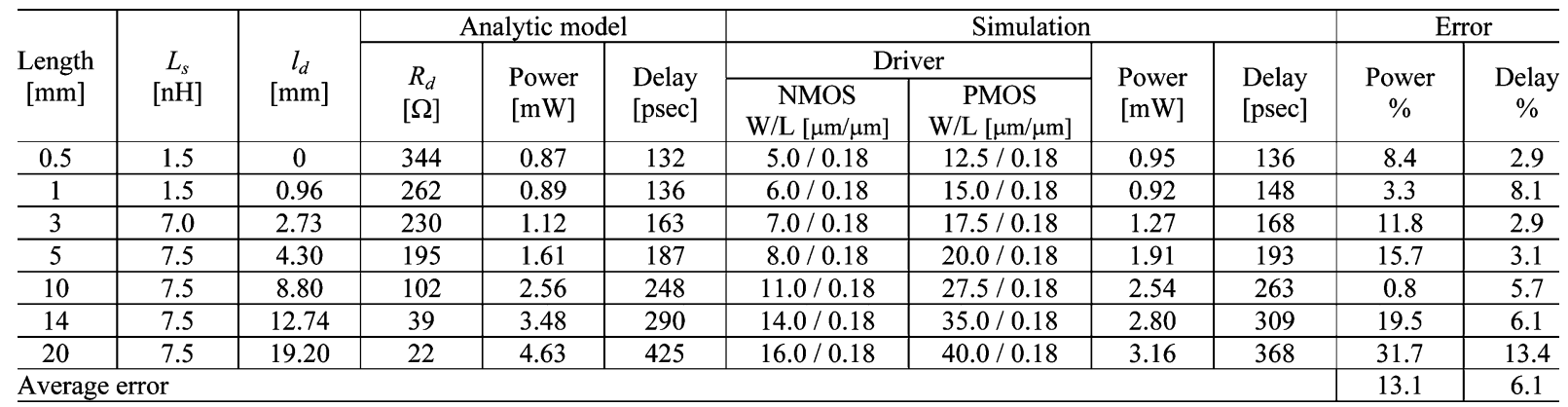

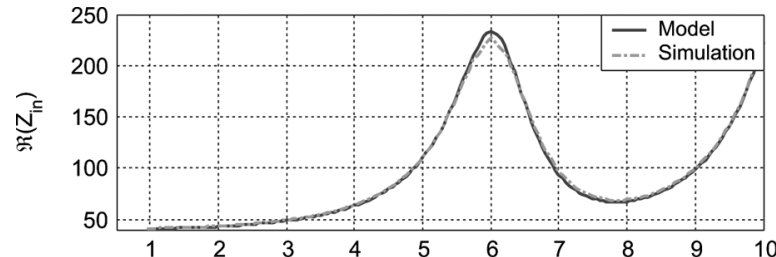

(a)

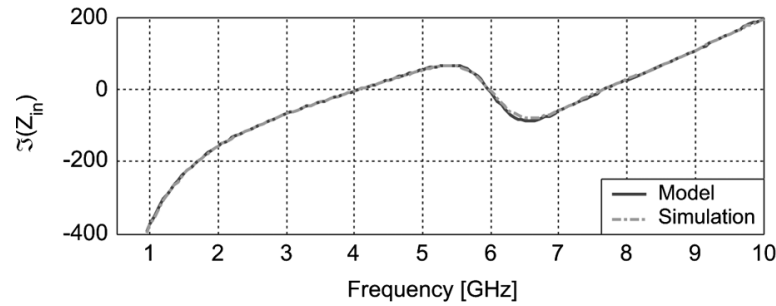

(b)

Fig. 14. QRN input impedance: (a) real part; (b) imaginary part.

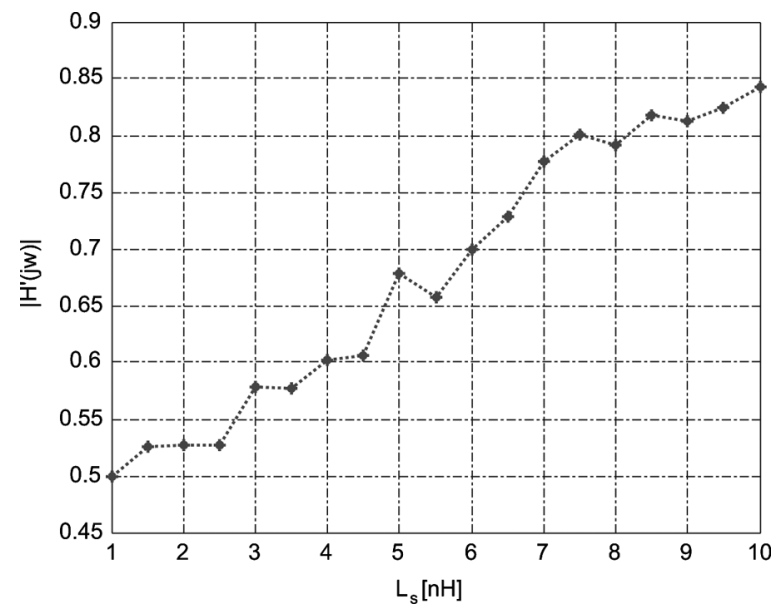

Fig. 15. Magnitude of the transfer function as a function of inductance at a $5-\mathrm{GHz}$ operating frequency. inductor. This behavior implies that a full swing voltage waveform is delivered at the far end only for this inductance.

The driver in this example is a $0.18-\mu \mathrm{m}$ CMOS inverter with $\mathrm{P} / \mathrm{N}=20 / 8$. The transmitter is based on the proposed circuit shown in Fig. 5(c), and the transistor widths are listed in Table I. The transmitter circuit load capacitance (i.e., the input capacitance of the driver) is 52.4 pf. The simulated input and output data signals described in the time domain are shown in Fig. 16. Note that the square data waveform is distributed to the far end, achieving a full rail-to-rail voltage swing. In this example, a "1000101111" bit stream is transmitted at $5 \mathrm{~Gb} / \mathrm{s}$. The simulated and analytic power consumption and delay of the receiver, transmitter, and QRN circuits are listed in Table II.

To evaluate the noise performance of the $\mathrm{QRN}$ for this case study, eye diagrams of nonresonant and quasi-resonant interconnects are generated by transmitting a random bit stream. To represent a noisy environment, the power supply and ground signals are corrupted by the addition of Gaussian noise, as shown in Fig. 17(a). The injected noise signal includes low frequencies to emulate the resonances produced by the package inductance and capacitance. From Fig. 17(c), observe that a large open eye is formed, exhibiting a high signal-to-noise ratio. The high noise rejection exhibited by the QRN is attributed to the detection and transmission of a single harmonic when a high logic level is transmitted. An eye diagram of an interconnect with repeaters is shown in Fig. 17(b). The jitter of the quasi-resonant interconnect (40 ps) is two times less than the nonresonant interconnect (80 ps).

\section{SimUlation ResUlTS AND COMPARISON}

To evaluate the accuracy of the model as compared to simulations, a $5 \mathrm{~Gb} / \mathrm{s}$ data signal and $0.5,1,3,5,10,14$, and $20 \mathrm{~mm}$ length interconnects are considered, as listed in Table III. Good agreement between simulation and the model is demonstrated, exhibiting an average error of $13.1 \%$ and $6.1 \%$, respectively, for 


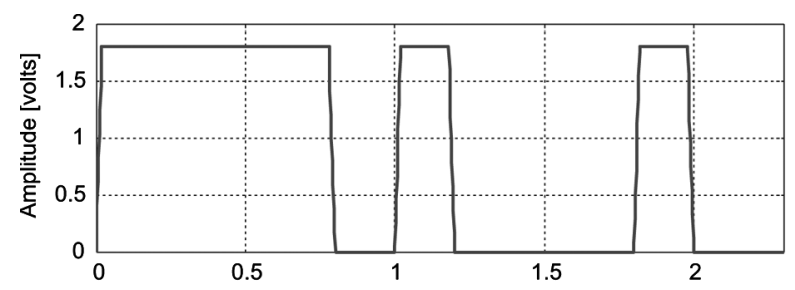

(a)

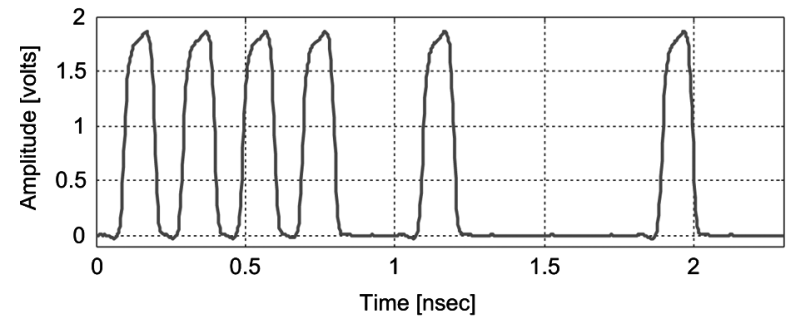

(a)

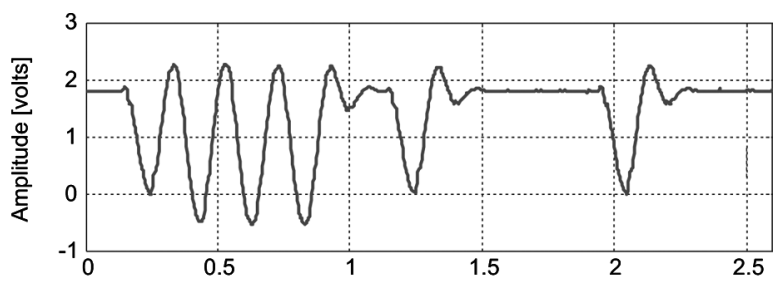

(c)

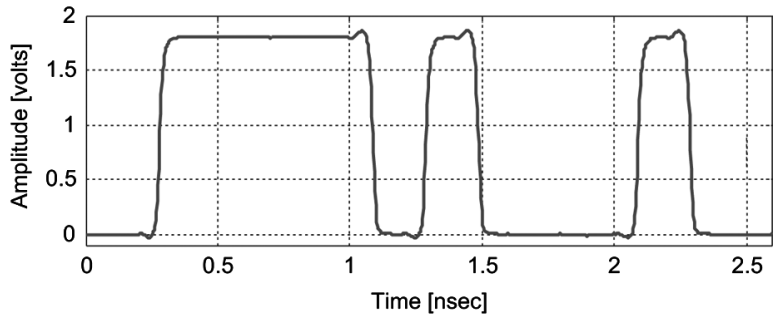

(d)

Fig. 16. Ten bit data stream example: (a) data at input of transmitter; (b) data at output of transmitter; (c) data at input of receiver; (d) data at output of receiver.
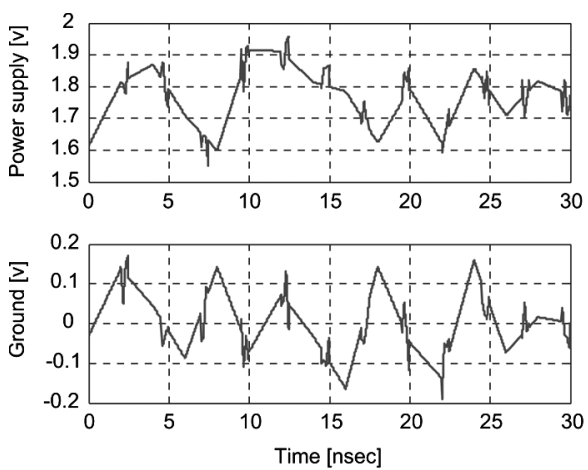

(a)

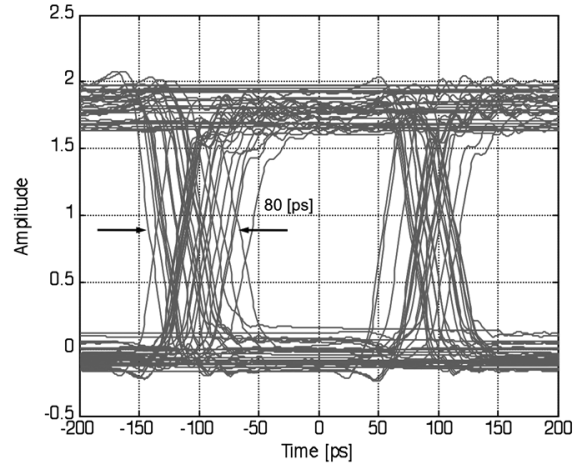

(b)

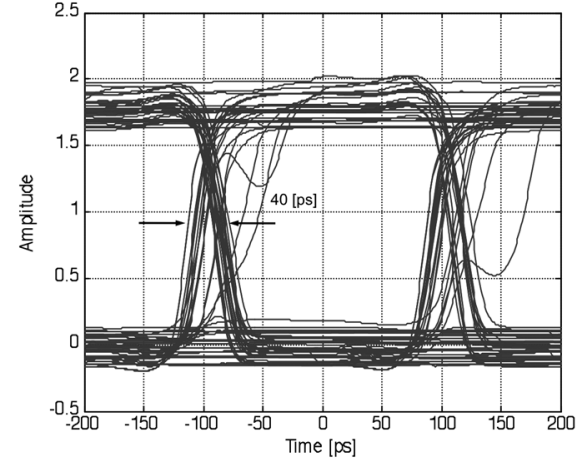

(c)

Fig. 17. Noise analysis: (a) noisy power supply and ground signals; (b) eye diagram of an interconnect with repeaters; (c) eye diagram of quasi-resonant interconnect.

TABLE IV

COMPARISON OF POWER CONSUMPTION AND DELAY

\begin{tabular}{c|c|c|c|c|c|c}
\hline \multirow{2}{*}{$\begin{array}{c}\text { Length } \\
{[\mathrm{mm}]}\end{array}$} & \multicolumn{2}{|c|}{ This work } & \multicolumn{2}{c|}{$\begin{array}{c}\text { Repeater } \\
\text { insertion }\end{array}$} & \multicolumn{2}{c}{ Improvement } \\
\cline { 2 - 7 } & $\begin{array}{c}\text { Power } \\
{[\mathrm{mW}]}\end{array}$ & $\begin{array}{c}\text { Delay } \\
{[\mathrm{ps}]}\end{array}$ & $\begin{array}{c}\text { Power } \\
{[\mathrm{mW}]}\end{array}$ & $\begin{array}{c}\text { Delay } \\
{[\mathrm{ps}]}\end{array}$ & $\begin{array}{c}\text { Power } \\
\%\end{array}$ & $\begin{array}{c}\text { Delay } \\
\%\end{array}$ \\
\hline 0.5 & 1.02 & 136 & 5.92 & 104 & 82.8 & -23.5 \\
\hline 1 & 0.99 & 148 & 10.79 & 160 & 90.8 & 7.5 \\
\hline 3 & 1.34 & 168 & 16.87 & 255 & 92.1 & 34.1 \\
\hline 5 & 1.98 & 193 & 25.20 & 368 & 92.1 & 47.5 \\
\hline 10 & 2.61 & 263 & 51.20 & 1100 & 94.9 & 76.1 \\
\hline
\end{tabular}

the power consumption and delay. The main reason for these discrepancies is due to the assumption of a linear driver model.

To evaluate the proposed methodology as compared to a traditional buffer insertion method, a $5 \mathrm{~Gb} / \mathrm{s}$ data signal and $0.5,1$, 3,5 , and $10 \mathrm{~mm}$ length interconnects are considered, as listed in Table IV. It is a challenge to design repeaters to drive long interconnects at frequencies as high as $5 \mathrm{GHz}$ in a $0.18-\mu \mathrm{m}$ CMOS technology. Hence, signal integrity in this repeater insertion case has been compromised for the sake of this comparison. From Table IV, the average reduction in power consumption and
TABLE V

PERFoRMANCE COMPARISON OF QUASI-RESONANT METHOD WITH DIFFERENT APPROACHES

\begin{tabular}{|c|c|c|c|c|c|}
\hline & $\begin{array}{c}\text { Technology } \\
\text { [nm] }\end{array}$ & $\begin{array}{c}\text { Speed } \\
{[\mathrm{Gbps}]}\end{array}$ & $\begin{array}{c}\text { Length } \\
{[\mathrm{mm}]}\end{array}$ & $\begin{array}{l}\text { Power } \\
{[\mathrm{mW}]}\end{array}$ & $\begin{array}{c}\text { Delay } \\
\text { [ps] }\end{array}$ \\
\hline This work & 180 & 5 & 3 & 1.34 & 168 \\
\hline Signal modulation [9] & 180 & 8 & 3 & 27.12 & 280 \\
\hline Improvement & & & & $20 \mathrm{X}$ & $40.0 \%$ \\
\hline This work & 180 & 5 & 20 & 3.23 & 368 \\
\hline Pulsed current [10] & 180 & 1 & 20 & 16 & 300 \\
\hline Improvement & & & & $5 \mathrm{X}$ & $-18.5 \%$ \\
\hline $\begin{array}{c}\text { This work } \\
\end{array}$ & 180 & 5 & 5 & 1.91 & 193 \\
\hline Optics (edge emitting) [11] & 250 & 3 & 5 & 78 & 260 \\
\hline Improvement & & & & $40 \mathrm{X}$ & $25.8 \%$ \\
\hline This work & 180 & 5 & 5 & 1.98 & 193 \\
\hline Optics (VCSEL) [11] & 250 & 3 & 5 & 66 & 300 \\
\hline Improvement & & & & $33 X$ & $35.6 \%$ \\
\hline This work & 180 & 5 & 14 & 2.8 & 309 \\
\hline Loss compensation [20] & 180 & 3 & 14 & 6 & 140 \\
\hline Improvement & & & & $2 X$ & $-54.7 \%$ \\
\hline
\end{tabular}

delay is $91.1 \%$ and $37.8 \%$, respectively. The performance improvement is due to the repeaterless nature of the quasi-resonant interconnect.

A comparison between the proposed methodology and other aggressive approaches described in the literature is listed in Table V. Interestingly, the greatest reduction in power consumption of $97.1 \%$ and delay of $35.6 \%$ occurs as compared to 
TABLE VI

AREA COMPARISON OF REPEATERS AND ON-CHIP SPIRAL INDUCTORS FOR DIFFERENT TECHNOLOGIES

\begin{tabular}{c|c|c|c|c|c|c}
\hline \multirow{2}{*}{$\begin{array}{l}\text { Length } \\
{[\mathrm{mm}]}\end{array}$} & \multicolumn{2}{|c|}{ Repeater insertion $\left[\mu \mathrm{m}^{2}\right]$} & \multicolumn{2}{c|}{ Quasi-resonant $\left[\mu \mathrm{m}^{2}\right]$} & \multicolumn{2}{c}{ Area overhead } \\
\cline { 2 - 7 } & $180[\mathrm{~nm}]$ & $50[\mathrm{~nm}]$ & $180[\mathrm{~nm}]$ & $50[\mathrm{~nm}]$ & $180[\mathrm{~nm}]$ & $50[\mathrm{~nm}]$ \\
\hline 0.5 & 19 & 57 & 9063 & 1007 & $480 \mathrm{X}$ & $17 \mathrm{X}$ \\
\hline 1 & 32 & 96 & 9063 & 1007 & $288 \mathrm{X}$ & $10 \mathrm{X}$ \\
\hline 3 & 44 & 132 & 6803 & 756 & $155 \mathrm{X}$ & $6 \mathrm{X}$ \\
\hline 5 & 63 & 189 & 6722 & 747 & $107 \mathrm{X}$ & $4 \mathrm{X}$ \\
\hline 10 & 126 & 378 & 6722 & 747 & $53 \mathrm{X}$ & $2 \mathrm{X}$ \\
\hline
\end{tabular}

optoelectronic links [11]. The power consumption overhead in [11] is due to the edge emitting laser modulator at the transmitting edge and the photodiode and signal level restorer at the receiving end of the optical link. This comparison suggests that novel signaling schemes incorporating electrical interconnects outperforms optoelectronic solutions. These results are obtained despite the optical link transmitting a slower signal (3 $\mathrm{Gb} / \mathrm{s})$ as compared to the resonant link (5 Gb/s).

With the exception of pulsed current [9], the resonant link transmits a higher frequency signal than the other methods. The proposed quasi-resonant interconnect methodology outperforms all of the other approaches described in the literature in power and, in most cases, in both power and latency. The primary tradeoff of the proposed methodology is the large area occupied by the on-chip inductor. This additional area is required to achieve a high $\mathrm{Q}$ inductor. In more advanced technologies, however, the area required for these inductors will be much lower. A comparison of the total area of repeaters and quasi-resonant methods for different technology nodes is listed in Table VI. The reduction in area overhead for a 50-nm CMOS technology is due to the requirement to insert additional repeaters to overcome the losses associated with long thin wires. Concurrently, the area of the on-chip inductor is lower due to the decrease in wire width and spacing.

\section{CONCLUSION}

A methodology is described in this paper for designing quasiresonant interconnect networks. An accurate model is presented based on transmission line theory and a lumped high frequency model of an on-chip spiral inductor. The accuracy of the model enables the design of low power, low latency resonant communication links. The methodology can be used to determine the specific inductance $L_{s}$, insertion point $l_{d}$, and driver resistance $R_{d}$ that minimizes the power-delay product.

Good agreement between the proposed model and simulation is exhibited, achieving an average error of $13.1 \%$ and $6.1 \%$ for the power consumption and delay, respectively. Quasi-resonant interconnects are shown to outperform other technologically aggressive circuit approaches. For buffered lines, an average reduction of $90.5 \%$ and $28.3 \%$ is obtained in power consumption and delay, respectively. As compared to optical links, a reduction of $97.0 \%$ and $35.6 \%$ is observed in power consumption and delay, respectively. These results show that quasi-resonant interconnects exhibit superior performance, suitable for high performance, high complexity integrated circuits.

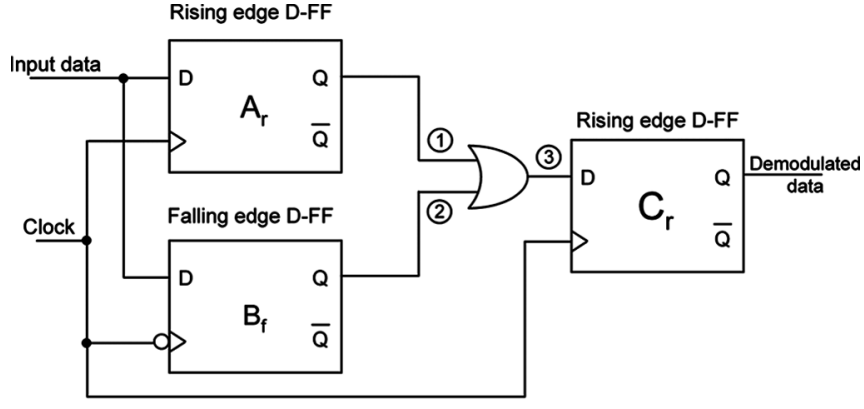

(a)

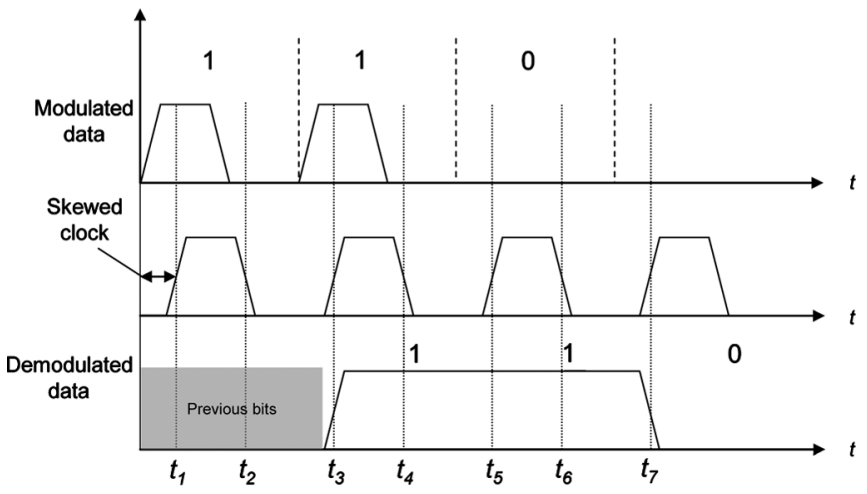

(b)

Fig. 18. Alternative receiver circuit: (a) gate implementation; (b) timing diagram.

\section{APPENDIX A \\ Alternative RECEIVER CIRCUIT}

The receiver circuit located at the far end of the interconnect (see Fig. 2) demodulates the transmitted data into the original signal. The proposed receiver circuit and timing diagram are depicted in Fig. 18.

The receiver circuit consists of three D flip-flops and an OR gate, as shown in Fig. 18(a). This circuit demodulates the received data regardless of the relative skew between the clock and the modulated signal. Note that $A_{r}$ and $C_{r}$ are rising edge flip-flops, and $B_{r}$ is a falling edge flip-flop.

As an example of the operation of the demodulation circuit, consider the timing diagram shown in Fig. 18(b). At time $t_{1}$ (rising edge of the clock), $A_{r}$ senses logic high of the input signal and transfers the signal to node 1. At this point, the output of the OR gate may not be correct. At time $t_{2}$ (falling edge of the clock), $B_{f}$ senses logic low and transfers the signal to node 2 . At this point, node 3 is logic high. On the next rising edge of the clock (time $t_{3}$ ), $C_{r}$ transfers a logic high signal at node 3 to the output. At the same time, $A_{r}$ senses the next modulated bit. If logic low is sensed by both $A_{r}$ and $B_{f}$, logic low is transferred 


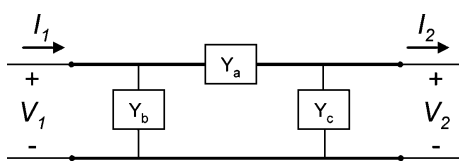

Fig. 19. Simplified admittance network for the spiral inductor model.

to the output (as demonstrated in the timing diagram depicted in Fig. 18(b) at time $t_{7}$ ). The demodulated signal at the output is synchronized with the clock. Note that the delay of the demodulation circuit is relative to the introduced skew. Also note that the skew can be either positive or negative.

\section{APPENDIX B \\ ABCD Matrices of THE QUASI-RESONANT NETWORK} is

The ABCD matrix for a transmission line as depicted in Fig. 7

$$
M_{i n t 1}=\left[\begin{array}{cc}
\cosh \gamma l & Z_{0} \sinh \gamma l \\
\frac{1}{Z_{0}} \sinh \gamma l & \cosh \gamma l
\end{array}\right]
$$

where $Z_{0}, \gamma$, and $l$ are the characteristic impedance, propagation constant, and line length, respectively. Similarly, $M_{\text {int2 }}$ is

$$
M_{\mathrm{int} 2}=\left[\begin{array}{cc}
\cosh \gamma\left(l-l_{d}\right) & Z_{0} \sinh \gamma\left(l-l_{d}\right) \\
\frac{1}{Z_{0}} \sinh \gamma\left(l-l_{d}\right) & \cosh \gamma\left(l-l_{d}\right)
\end{array}\right] .
$$

To derive the $A B C D$ matrix $M_{s}$, the spiral inductor depicted in Fig. 3(b) is simplified into the network shown in Fig. 19.

From this network, the $A B C D$ matrix is

$$
M_{s}=\left[\begin{array}{cc}
1+\frac{Y_{c}}{Y_{a}} & \frac{1}{Y_{a}} \\
Y_{b}+Y_{c}+\frac{Y_{b} Y_{c}}{Y_{a}} & 1+\frac{Y_{1}}{Y_{3}}
\end{array}\right]
$$

where

$$
\begin{aligned}
Y_{a} & =\frac{1}{j \omega L_{\mathrm{series}}+\frac{R_{\mathrm{ac}}\left(R_{\text {skin }}+j \omega L_{\mathrm{skin}}\right)}{R_{\mathrm{ac}}+R_{\mathrm{skin}}+j \omega L_{\mathrm{skin}}}}+j \omega C_{p} \\
Y_{b} & =\frac{1}{\frac{1}{\left(j \omega C_{\mathrm{ox} 1}\right)}+\frac{\left(\frac{R_{\mathrm{sub} 1}}{j \omega C_{\mathrm{sub} 1}}\right)}{\left(\frac{R_{\mathrm{sub} 1}+1}{j \omega C_{\mathrm{sub} 1}}\right)}} \\
Y_{c} & =\frac{1}{\frac{1}{\left(j \omega C_{\mathrm{ox} 2}\right)}+\frac{\left(\frac{R_{\mathrm{sub} 2}}{j \omega C_{\mathrm{sub} 2}}\right)}{\left(\frac{R_{\mathrm{sub} 2}+1}{j \omega C_{\mathrm{sub} 2}}\right)}} .
\end{aligned}
$$

Finally, the $A B C D$ matrix of the capacitive load is

$$
M_{l}=\left[\begin{array}{cc}
1 & 0 \\
j \omega C_{l} & 1
\end{array}\right] .
$$

\section{APPENDIX C}

\section{DERIVATION OF THE QUASI-RESONANT NETWORK DELAY EXPRESSION}

The transfer function of the QRN at each angular frequency can be represented as

$$
H^{\prime}(j \omega)=\left|H^{\prime}(\omega)\right| e^{j \beta(\omega)}
$$

where $\beta(\omega)$ is the phase of the transfer function. The output waveform in the time domain for a linear system, described by a transfer function such as (B1) and stimulated by a periodic square signal, can be approximated by

$$
V_{\text {out }}(t) \approx \frac{V_{\mathrm{dd}}}{2}+A_{1} \sin \left(\omega_{p} t+\phi_{1}\right)+A_{3} \sin \left(3 \omega_{p} t+\phi_{3}\right)
$$

where

$$
A_{m=1,3}=\frac{2 t_{p} V_{\mathrm{dd}}}{t_{r} m^{2} \pi^{2}}\left|\sin \left(\frac{m \omega_{p} t_{r}}{2}\right)\right|\left|H^{\prime}\left(m \omega_{p}\right)\right|
$$

and

$$
\phi_{m=1,3}=-\frac{m \omega_{p} t_{r}}{2}+\beta\left(m \omega_{p}\right)
$$

Note that the approximated closed-form solution in (B2) is available only when considering the first three harmonics of the input signal. To determine the 50\% delay, (B2) is set to $V_{\mathrm{dd}} / 2$. A third-order trigonometric expression can be obtained as

$$
a_{3} x^{3}+a_{2} x^{2}+a_{1} x+a_{0}=0
$$

where $x=\tan \left(\omega_{p} t\right)$ and

$$
\begin{aligned}
& a_{0}=A_{1} \sin \phi_{1}+A_{3} \sin \phi_{3} \\
& a_{1}=A_{1} \cos \phi_{1}+3 A_{3} \cos \phi_{3} \\
& a_{2}=A_{1} \sin \phi_{1}-3 A_{3} \sin \phi_{3} \\
& a_{3}=A_{1} \cos \phi_{1}-A_{3} \cos \phi_{3} .
\end{aligned}
$$

A third order polynomial such as (B5) has either one or three real roots, and a closed-form solution exists. In the case of a single real root $x_{0}$, the $50 \%$ delay can be calculated from (18).

\section{ACKNOWLEDGMENT}

The authors would like to thank the reviewers for their educational and constructive comments and suggestions. They would also like to acknowledge one of the reviewers in particular for his discussion regarding Pupin coils. The reviewers' suggestions enabled the authors to significantly improve this paper.

\section{REFERENCES}

[1] ITRS, "The International Technology Roadmap for Semiconductors (ITRS)," 2005.

[2] Y. I. Ismail, E. G. Friedman, and J. L. Neves, "Figures of merit to characterize the importance of on-chip inductance," IEEE Trans. Very Large Scale Integr. (VLSI) Syst., vol. 7, no. 6, pp. 442-449, Dec. 1999.

[3] Y. I. Ismail and E. G. Friedman, "Effects of inductance on the propagation delay and repeater insertion in VLSI circuits," IEEE Trans. Very Large Scale Integr. (VLSI) Syst., vol. 8, no. 2, pp. 195-206, Apr. 2000

[4] A. Valentian and A. Amara, "On-chip signaling for ultra low-voltage $0.13 \mu \mathrm{m}$ CMOS SOI technology," in Proc. IEEE Northeast Workshop Circuits Syst., Jun. 2004, pp. 169-172.

[5] N. Tzartzanis and W. W. Walker, "Differential current-mode sensing for efficient on-chip global signaling," IEEE J. Solid-State Circuits, vol. 40, no. 11, pp. 2141-2147, Nov. 2005.

[6] R. Bashirullah, W. Liu, R. Cavin, and D. Edwards, "A hybrid current/voltage mode on-chip signaling scheme with adaptive bandwidth capability," IEEE Trans. Very Large Scale Integr. (VLSI) Syst., vol. 12, no. 8, pp. 876-880, Aug. 2004.

[7] I. Ben Dhaou, V. Sundararajan, H. Tenhunen, and K. K. Parhi, "Energy efficient signaling in deep submicron CMOS technology," in Proc. IEEE Int. Symp. Quality Electron. Des., Mar. 2001, pp. 319-324. 
[8] V. Venkatraman, M. Anders, H. Kaul, W. Burleson, and R. Krishnamurthy, "A low-swing signaling technique for $65 \mathrm{~nm}$ on-chip interconnects," in Proc. IEEE Int. SoC Conf., Sep. 2006, pp. 289-292.

[9] R. T. Chang, N. Talwalker, C. P. Yue, and S. S. Wong, "Near speed-oflight signaling over on-chip electrical interconnects," IEEE J. SolidState Circuits, vol. 38, no. 5, pp. 834-838, May 2003.

[10] A. P. Jose, G. Patounakis, and K. L. Shepard, "Near speed-of-light on-chip interconnects using pulsed current-mode signaling," in Proc. IEEE Symp. VLSI Circuits, Jun. 2005, pp. 108-111.

[11] E. D. Kyriakis-Bitzaros, N. Haralabidis, M. Lagadas, A. Georgakilas, Y. Moisiadis, and G. Halkias, "Realistic end-to-end simulation of the optoelectronic links and comparison with the electrical interconnections for system-on-chip applications," IEEE J. Lightw. Technol., vol. 19, no. 10 , pp. $1532-1542$, Oct. 2001.

[12] M. I. Pupin, "Propagation of long electrical waves," Amer. Inst. Elect. Eng. Trans., vol. XV, pp. 93-142, Mar. 1899.

[13] M. I. Pupin, "Art of reducing attenuation of electrical waves and apparatus therefore," U.S. Patent No. 652 230, Jun. 19, 1900.

[14] A. Marinčić, "Analysis of transmission line with periodical inductance loading," Microw. Rev., vol. 10, no. 2, pp. 43-48, Nov. 2004.

[15] V. L. Chi, "Salphasic distribution of clock signals for synchronous systems," IEEE Trans. Comput., vol. 43, no. 5, pp. 597-602, May 1994.

[16] S. C. Chan, K. L. Shepard, and P. J. Restle, "Design of resonant global clock distributions," in Proc. IEEE Int. Conf. Comput. Des., Oct. 2003, pp. $248-253$.

[17] J. Chueh, M. C. Papaefthymiou, and C. H. Ziesler, "Two-phase resonant clock distribution," in Proc. IEEE Ann. Symp. VLSI, May 2005, pp. $65-70$.

[18] J. Rosenfeld and E. G. Friedman, "Design methodology for global resonant H-Tree clock distribution networks," IEEE Trans. Very Large Scale Integr. (VLSI) Syst., vol. 15, no. 2, pp. 135-148, Feb. 2007.

[19] A. P. Jose and K. L. Shepard, "Distributed loss compensation for lowlatency on-chip interconnects," in Proc. IEEE Int. Solid-State Circuits Conf., Feb. 2006, pp. 392-393.

[20] M. E. Van Valkenburg, Network Analysis. Englewood Cliffs, NJ: Prentice-Hall, 1974

[21] G. Chen and E. G. Friedman, "An RLC interconnect model based on Fourier analysis," IEEE Trans. Comput.-Aided Des. Integr. Circuits Syst., vol. 24, no. 2, pp. 170-183, Feb. 2005.

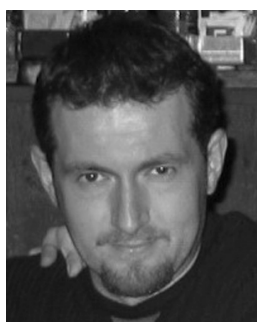

Jonathan Rosenfeld received the B.S. degree in mechanical engineering from Technion-Israel Institute of Technology, Haifa, Israel, in 1999, the Bachelors of Technology degree (with honors) in electronic engineering from Ort-Braude College, Karmiel, Israel, in 2003, and the M.S. degree in electrical and computer engineering from the University of Rochester, Rochester, NY, in 2005, where he is currently pursuing the $\mathrm{Ph} . \mathrm{D}$. degree in electrical and computer engineering.

He was an intern with Intrinsix Corporation, Fairport, NY, in 2005, where he designed Gm-C circuits for a $\Sigma \Delta$-modulator ADC for an FM tuner. In 2007, he was an intern with Eastman Kodak Company, Rochester, NY, where he developed column-based multiple ramp integrated ADC for CMOS image sensors. His research interests include the areas of interconnect design, resonant clock and data distribution networks, on-chip inductive effects, and the design of analog and mixed-signal integrated circuits.

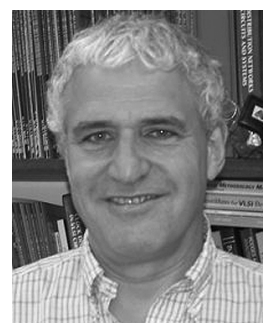

Eby G. Friedman (F'00) received the B.S. degree from Lafayette College, Easton, PA, in 1979, and the M.S. and Ph.D. degrees from the University of California, Irvine, in 1981 and 1989, respectively, all in electrical engineering.

From 1979 to 1991, he was with Hughes Aircraft Company, rising to the position of manager of the Signal Processing Design and Test Department, where he was responsible for the design and test of high performance digital and analog ICs. He has been with the Department of Electrical and Compute Engineering, University of Rochester, Rochester, NY, since 1991, where he is a Distinguished Professor, the Director of the High Performance VLSI/IC Design and Analysis Laboratory, and the Director of the Center for Electronic Imaging Systems. He is also a Visiting Professor with the Technion-Israel Institute of Technology, Haifa, Israel. His current research and teaching interests include high performance synchronous digital and mixed-signal microelectronic design and analysis with application to high speed portable processors and low power wireless communications. He is the author of more than 300 papers and book chapters, several patents, and the author or editor of 10 books in the fields of high speed and low power CMOS design techniques, high speed interconnect, and the theory and application of synchronous clock and power distribution networks.

Dr. Friedman is the Regional Editor of the Journal of Circuits Systems and Computers, a Member of the editorial boards of the Analog Integrated Circuits and Signal Processing, Microelectronics Journal, Journal of Low Power Electronics, and Journal of VLSI Signal Processing, Chair of the IEEE TRANSACTIONS ON VERY LARGE SCALE INTEGRATION (VLSI) SYSTEMS steering committee, and a Member of the technical program committee of a number of conferences. He previously was the Editor-in-Chief of the IEEE TRANSACTIONS ON Very LARGE SCALE INTEgRation (VLSI) Systems, a Member of the editorial board of the Proceedings of the IEEE and the IEEE Transactions on Circuits and Systems II: ANALOG and Digital Signal ProCESSING, a Member of the Circuits and Systems (CAS) Society Board of Governors, CAS liaison to the Solid-State Circuits Society, Program and Technical chair of several IEEE conferences, Guest Editor of several special issues in a variety of journals, and a recipient of the Howard Hughes Masters and Doctoral Fellowships, an IBM University Research Award, an Outstanding IEEE Chapter Chairman Award, the University of Rochester Graduate Teaching Award, and a College of Engineering Teaching Excellence Award. He is a Senior Fulbright Fellow. 